\author{
Haruhiko MARUYAMA ${ }^{1) *}$, Haruki HOSOE ${ }^{1)}$, Kota NAGAMATSU ${ }^{2)}$, Rui KANO ${ }^{1)}$ and \\ Hiroshi KAMATA ${ }^{1)}$
}

1)Laboratory of Veterinary Clinical Pathology, Department of Veterinary Medicine, Nihon University, 1866 Kameino, Fujisawa, Kanagawa 252-0880, Japan

2) Nagamatsu Animal Hospital, 2-3-10 Nishiku Sakaisunayama, Niigata 950-2044, Japan

J. Vet. Med. Sci.

79(5): 822-826, 2017

doi: 10.1292/jvms.16-0602

Received: 21 November 2016

Accepted: 20 March 2017

Published online in J-STAGE: 7 April 2017
ABSTRACT. The feline $F 12$ gene was examined to identify a mutation associated with coagulation factor XII (FXII) deficiency in a litter of 6 cats, including 2 cats with severely reduced FXII activity (7.1 and 9.3\%, respectively) and 4 cats with moderately reduced FXII activity (range 36.0 to $46.3 \%$ ). Cats with severely reduced FXII activity were homozygous for a $\mathrm{G}$ to $\mathrm{C}$ missense mutation in exon 13 of the $F 12$ gene, resulting in an amino acid change (p.G544A). Cats with moderately reduced FXII activity were heterozygous for this mutation. Expression studies revealed reduced secretion of p.G544A mutant FXII protein from transfected HEK293 cells compared with wild type FXII. These results reveal a novel F12 mutation in FXII deficient cats and define the underlying mechanism for low FXII activity in homozygotes.

KEY WORDS: cat, factor XII, mutation

Factor XII (FXII) is a contact pathway coagulation factor whose reported biologic activities include the initiation of the intrinsic pathway of blood coagulation, the activation of the kallikrein-kinin system, the generation of bradykinin and modulation of vascular permeability [11]. The feline F12 gene consists of 14 exons and 13 introns, and encodes 609 amino acids with all the structural domains reported in human beings: a leader peptide; a fibronectin domain type II; an epidermal-growth-factor-like (EGF-like) domain; a fibronectin domain type I; a second EGF-like domain; a kringle domain; a proline-rich region and the catalytic domain [1, 13].

Feline FXII deficiency is a common hereditary trait with autosomal recessive inheritance pattern [2, 7]. This deficiency manifests as prolonged clotting time in the activated partial thromboplastin time (APTT) screening test, with no signs of abnormal bleeding [2]. Bender et al. described a single base deletion in exon 11 of the feline $F 12$ gene (c.1321 delC) that caused a frame shift and missense mutation at L441C (C441 fsX119) with a premature stop codon at position 560 in the amino acid sequence. The resultant truncated FXII protein lacked most of catalytic domain's active site [1]. Here, we report that a novel missense mutation of feline F12 gene, found in a litter of domestic short hair (DSH) cats, caused FXII deficiency due to decreased secretion of FXII protein from cells.

The 2 index cases were from a litter of 7-month-old DSH cats, including 3 males and 3 females, brought to the Nagamatsu Animal Hospital for castration or spaying. The owners reported no prior clinical problems or bleeding tendencies for any of the cats, and no abnormalities were found on physical examination. However, on pre-operative coagulation screening tests, 2 female cats had extremely prolonged APTTs (case A; $86.7 \mathrm{sec}$ and case B; $93.6 \mathrm{sec}$, reference range; 20.0-42.0 sec). These $2 \mathrm{cats}$ had normal prothrombin times (PT) (case A; $9.7 \mathrm{sec}$ and case B; $9.8 \mathrm{sec}$, reference range; $9.3-11.3 \mathrm{sec}$ ), and the coagulation tests of the remaining 4 littermates were within reference range (Table 1). The coagulation screening tests were performed by COAG2V (Wako Pure Chemical Industries, Osaka, Japan).

To further define the cause of the 2 female siblings' prolonged APTT, intrinsic coagulation factors activities (Factors VIII, IX, $\mathrm{XI}, \mathrm{XII}$ and prekallikrein), were assayed at our laboratory. In one-stage clotting time tests, citrate-anticoagulated plasmas were diluted 5-fold with Veronal buffer (Sysmex, Kobe, Japan), and then, human factor-deficient plasmas (George King Bio-Medical, Overland Park, KS, U.S.A.) were added to the diluted plasmas and APTT-based clotting times were measured by semi-automated coagulation analyzer, Amelung coagulometer KC4A (Heinrich Amelung GmbH, Lemgo, Germany) [9]. The activities of each intrinsic coagulation factor were calculated based on a standard curve derived from dilutions of feline pooled plasma, which was prepared by combining citrated plasmas collected from five clinically healthy cats.

The results of intrinsic coagulation factor activities are summarized in Table 1. Markedly decreased FXII activities (Case A:

*Correspondence to: Maruyama, H., Laboratory of Veterinary Clinical Pathology, Department of Veterinary Medicine, Nihon University, 1866 Kameino, Fujisawa, Kanagawa 252-0880, Japan. e-mail: maruyama.haruhiko@nihon-u.ac.jp

(C2017 The Japanese Society of Veterinary Science

This is an open-access article distributed under the terms of the Creative Commons Attribution Non-Commercial No Derivatives (by-ncnd) License. (CC-BY-NC-ND 4.0: https://creativecommons.org/licenses/by-nc-nd/4.0/) 
Table 1. Results of coagulation assays

\begin{tabular}{ccccrcccc}
\hline \multirow{2}{*}{ Case } & \multirow{2}{*}{ sex } & \multirow{2}{*}{ APTT $(\mathrm{sec})$} & \multirow{2}{*}{ PT $(\mathrm{sec})$} & \multicolumn{5}{c}{ Coagulation factor assays (\%) } \\
\cline { 5 - 8 } & & & FXII & FVIII & FIX & FXI & PK \\
\hline A & F & 86.7 & 9.7 & 7.1 & 140.1 & 168.4 & 223.2 & 393.1 \\
B & F & 93.6 & 9.8 & 9.3 & 180.1 & 205.4 & 243.7 & 410.3 \\
C & F & 21.7 & 9.7 & 46.3 & 191.7 & 195.7 & 241.7 & 426.0 \\
D & M & 23.2 & 9.9 & 41.8 & 274.9 & 203.4 & 194.0 & 634.9 \\
E & M & 32.7 & 9.5 & 36.0 & 292.8 & 217.8 & 214.6 & 449.6 \\
F & M & 34.5 & 9.7 & 42.4 & 286.7 & 207.4 & 230.4 & 403.8 \\
\hline
\end{tabular}

APTT, activated partial thromboplastin time; PT, prothrombin time; FXII, Factor XII coagulant activity; FVIII, Factor VIII coagulant activity; FIX, Factor IX coagulant activity; FXI, Factor XI coagulant activity; PK, prekallikrein activity.

Table 2. Primer pairs used for amplification of feline $F 12$ genomic DNA

\begin{tabular}{llllc}
\hline Amplified exon no. & Primer name & Nucleotide sequence (5' to 3') & $\begin{array}{c}\text { Primer position on feline } \\
\text { chromosome A1 a) }\end{array}$ & $\begin{array}{c}\text { Amlicon size } \\
\text { (bp) }\end{array}$ \\
\hline 1,2 & fFXII 1/2 F & GCTCGACCAATCTCTAATTC & $173,542,820-173,542,839$ & 696 \\
& fFXII 1/2 R & GTGAAGAATGTCCCGGATCC & $173,543,496-173,543,515$ & \\
\hline $3-8$ & fFXII 3/4 F & GAACCTCTTGAAGGCATAAC & $173,547,099-173,547,118$ & 1,804 \\
& fFXII 7/8R & GATTCCTGTAACCACCGGAG & $173,548,883-173,548,902$ & \\
\hline $9-12$ & fFXII 9/10 F & GACCATGCCTTCTGCAGGTG & $173,548,788-173,548,807$ & 1,382 \\
& fFXII 11/12 R & GAGCTGACTGCAGACACAAG & $173,550,150-173,550,169$ & \\
\hline 13,14 & fFXII 13/14 F & GTTCCAAAGCACTCCTGCTC & $173,550,377-173,550,396$ & 727 \\
& fFXII 13/14 R & GAGTGCCTACTCTATGCCTG & $173,551,084-173,551,103$ & \\
\hline
\end{tabular}

a) Felis catus isolate Cinnamon breed Abyssinian chromosome A1, Felis_catus_8.0, whole genome shotgun sequence (Accession No. NC_018723.2).

7.1\% and B: $9.3 \%$, respectively) were observed in the two cats with prolonged APTT. Furthermore, the remaining 4 littermates with normal APTT had FXII activities of approximately $40 \%$ of the pooled normal feline plasma. No other decreased factor activities were observed. Based on these results, the 2 cases with prolonged APTT were diagnosed with FXII deficiency, and the other 4 cases with normal APTT were considered likely carriers of the trait. After diagnosis of FXII deficiency as the cause of prolonged APTT for the 2 female siblings, all cats subsequently underwent castration or spaying without incident. None of the cats experienced abnormal bleeding.

We then performed molecular analyses to identify a F12 gene mutation associated with FXII deficiency in these cats. Genomic DNAs were extracted from the cell fractions remaining from the citrated blood submitted for coagulation factor assays, using QIAamp DNA Blood Mini kit (Qiagen, Hilden, Germany). Four primer pairs were constructed to amplify all exons and exon-intron junctions of feline F12 (Table 2). Polymerase chain reaction (PCR) was performed using PrimeSTAR Max DNA Polymerase (Takara-Bio, Otsu, Japan) and the following conditions; 35 cycles at $98^{\circ} \mathrm{C} 10 \mathrm{sec}, 55^{\circ} \mathrm{C} 5 \mathrm{sec}$ and $72^{\circ} \mathrm{C} 30 \mathrm{sec}$. The amplified PCR products were purified using Wizard SV Gel and PCR Clean-Up system (Promega, Madison, WI, U.S.A.) and then were processed for sequencing reactions using the Big Dye Terminator v3.1 Cycle Sequencing kit (Applied Biosystems, Foster City, CA, U.S.A.). The nucleotide sequences were determined by the direct sequencing method using ABI 3130 (Applied Biosystems).

Because several base differences between the 2 GenBank mRNA sequences (accession no. NM_001168212.2 and GQ981174.2) and the sequence of our cases were observed, we compared the sequences of the 2 affected cats with their carrier siblings. As a result, two single-base substitutions were found. One substitution was that guanine was replaced by cytosine in exon 13 (c.1631G>C), leading to a change in amino acid 544 from glycine to alanine (p.G544A) in the catalytic domain (Fig. 1). This mutation nucleotide sequence data was submitted to DNA Data Bank of Japan (accession number LC195886). This mutation was observed as homozygous in the 2 FXII deficient cats, but as heterozygous in their 4 siblings. The other substitution, a synonymous base change from $\mathrm{C}$ to $\mathrm{T}$ in exon 4, was not considered likely to influence FXII expression and was not further studied.

To further define the effect of the newly identified F12 mutation on FXII protein expression, we performed transient expression of recombinant feline FXII (rfFXII) protein using mammalian cells for western blot analysis. Feline liver total RNA was used for cDNA synthesis using PrimeScript II 1st strand cDNA Synthesis Kit (Takara-Bio). PCR for amplification of the full length feline FXII protein coding region of cDNA was performed using a primer pair (5'-CACCATGAGGGCTCTCCTGTTC-3' and 5'-GGAATTGGTGTGCTCCTGGATC-3'), feline liver cDNA and PrimeSTAR MAX DNA Polymerase (Takara-Bio). The amplicon was ligated into pcDNA 3.1D/V5-His TOPO plasmid vector, which could express fusion protein with V5-tag at the C-terminal (Invitrogen, Carlsbad, CA, U.S.A.). The constructed plasmid DNA vector was used to transform competent TOP 10 cells (Invitrogen). The constructed plasmid DNA Vector was extracted from the bacteria cultured in Luria-Bertani broth using NucleoBond Xtra Midi Plus EF (Macherey-Nagel, Duren, Germany). The sequence of the constructed plasmid vector was then compared with that of $F 12$ genomic sequence obtained from this study. We found a nonsynonymous nucleotide substitution in the FXII coding region of the constructed plasmid DNA vector. Therefore, to eliminate any influence of this substitution on the 
Wild

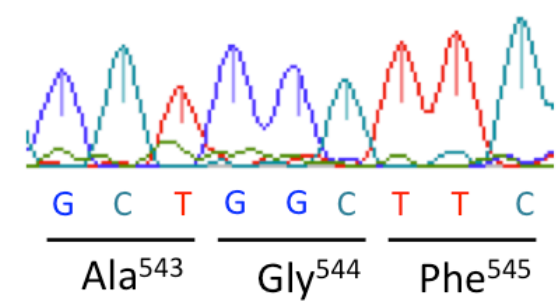

Affected

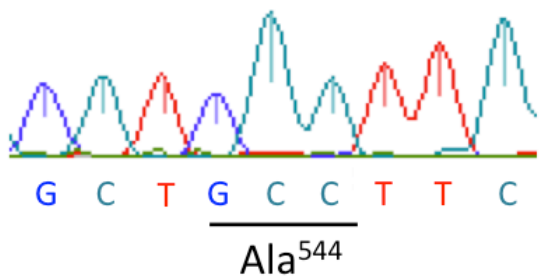

Carrier

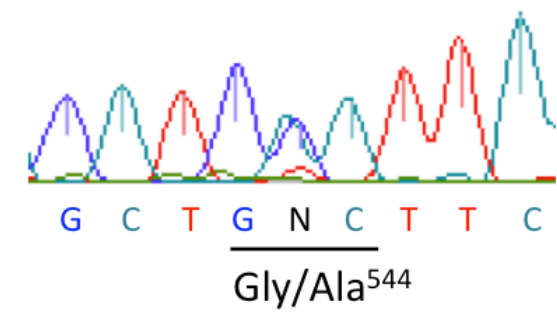

Fig. 1. Sequence analysis of feline F12 gene focusing on the mutation in exon 13 . The codon including nucleotide 1631 of wild type is glycine. The $\mathrm{G}$ to $\mathrm{C}$ missense mutation of affected cats leads to amino acid at 544 changing from glycine to alanine. In carrier, sequence waves of $\mathrm{G}$ and $\mathrm{C}$ were overlapped.

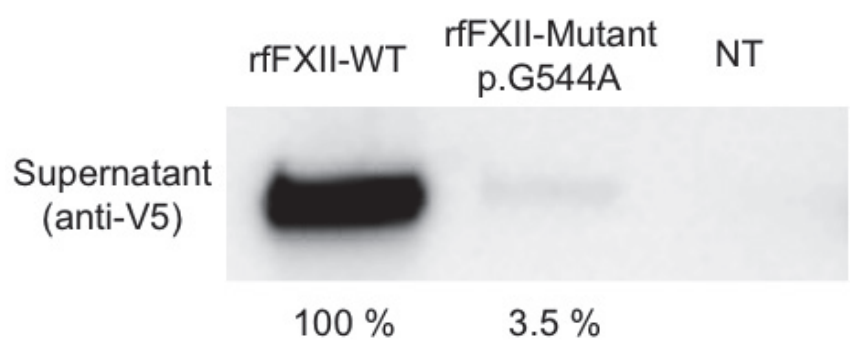

Cell lysate

(anti-V5)

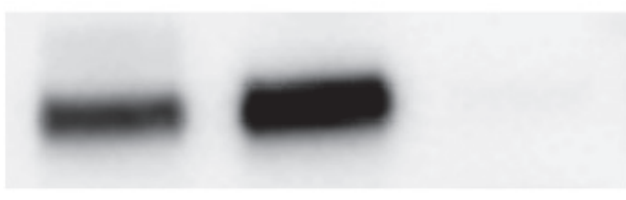

$100 \%$

$206.5 \%$

\section{Cell lysate}

(anti- $\beta$-actin)

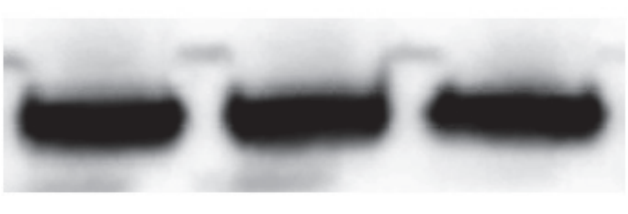

Fig. 2. rfFXII expression in supernatants and cells. The expression level of wild type rfFXII protein was assigned as $100 \%$. The mean value of the p.G544A rfFXII protein in the supernatants was 3.5\%, but $206.5 \%$ in the cell lysates. Anti-V5 monoclonal antibody is used for detecting rfFXII fused with V5-tag (upper and middle). Anti- $\beta$ actin monoclonal antibody is used for detecting $\beta$-actin as a loading control (lower). rfFXII: recombinant feline factor XII. WT: wild type. NT: no-template.

expression pattern of FXII protein, the nucleotide was replaced by the nucleotide corresponding to the genomic DNA sequence using a PrimeSTAR Mutagenesis Basal Kit (Takara-Bio) according to manufacturer's instruction. The genomic sequence-matched plasmid DNA vector was re-cloned and then used as the wild-type expression vector (accession number LC195885). The p.G544A mutant protein expression plasmid DNA vector was constructed from the wild-type expression plasmid DNA vector using PrimeSTAR Mutagenesis Basal Kit (Takara-Bio). The HEK293 cells were transfected with the wild-type or mutant plasmid DNA vector constructs using X-tremeGENE HP DNA Transfection (Roche, Mannheim, Germany) according to manufacturer's instructions. At $4 \mathrm{hr}$ after transfection, the culture media were replaced from Dulbecco's modified Eagle's medium supplemented with $10 \%$ fetal bovine serum to serum-free Opti-MEM (Invitrogen). The supernatants and cells were collected after $48 \mathrm{hr}$ of transfection. The collected cells were washed twice with phosphate-buffered saline and then lysed using CelLytic M (SignaAldrich, St. Louis, MO, U.S.A.). The lysed cells were centrifuged to pellet the cell debris, and the supernatants were used as cell lysate. The supernatants and cell lysates were stored at $-80^{\circ} \mathrm{C}$ until Western blot analyses. A "no-template" inserted pcDNA 3.1D/ V5-His TOPO plasmid DNA vector was used as a negative control.

To confirm the difference of expression levels between wild type and mutant rfFXII proteins, western blot experiments were performed. The supernatants and cell lysates were mixed with sodium dodecyl sulfate (SDS) sample buffer (58 $\mathrm{mM} \mathrm{Tris-Cl} \mathrm{pH}$ $6.8,1.7 \%$ SDS, $5 \%$ glycerol, $1.5 \%$ dithiothreitol and $0.02 \%$ bromophenol blue), respectively. These samples were electrophoresed on 4-20\% SDS-polyacrylamide gel and transferred onto a polyvinylidene difluoride membrane (Bio-Rad, Hercules, CA, U.S.A.). After blocking using Block Ace (DS Pharma Biomedical, Osaka, Japan), the membrane was incubated with peroxidase-labeled anti-V5 monoclonal antibody (Invitrogen) for detecting V5-taged rfFXII in supernatants and cell lysates or with peroxidaselabeled anti- $\beta$-actin monoclonal mouse antibody (Abcam, Cambridge, U.K.) for detecting $\beta$-actin as a loading control of cell lysates. Chemiluminescent detection was performed using ECL prime Western Blotting Detection Reagent (GE Healthcare, Buckinghamshire, U.K.) by ImageQuant LAS 400 mini (GE Healthcare). Mann-Whitney $U$ test was used for comparing the rfFXII protein expression levels between wild type and mutation type in western blot analysis. Values of $P<0.05$ were considered statistically significant.

In western blot analyses, the rfFXII protein was observed as an approximately $80 \mathrm{kDa}$ band detected by the anti-V5 monoclonal antibody. The expression level of rfFXII wild type protein was assigned a value of $100 \%$. In comparison, expression of p.G544A rfFXII protein in the transfected cell supernatants was markedly decreased (mean $\pm \mathrm{SD} ; 3.5 \pm 2.0 \%, P<0.05$ ), but significantly increased in cell lysates $(206.5 \pm 63.1 \%, P<0.05)$ (Fig. 2). 
In the present study, the novel mutation, c. $1631 \mathrm{G}>\mathrm{C}$, was found by comparing the sequence of feline $F 12$ gene of 6 littermates that included 2 siblings with severe FXII deficiency. The cats with severe FXII deficiency were homozygous for this mutation, and the cats with moderate reduction in FXII activity were heterozygous. Thus, the genotypes for this mutation were compatible with observed phenotypes. This novel mutation resulted in an amino acid substitution (p.G544A) and marked reduction in the amount of rfFXII protein secreted into transfected cell supernatants compared with that of wild-type transfectants. Notably, the c.1321delC, previously reported in DSH in the United States [1], was absent from all cats evaluated in our present study (Data not shown). The novel c. $1631 \mathrm{G}>\mathrm{C}$ mutation is therefore considered causative for FXII deficiency in the littermate DSH we studied.

In human FXII deficiency, a mutation which leads to amino acid change from glycine to glutamic acid at codon 531 (p.G531E) has been reported [5]. This mutation corresponds to the position of the feline p.G544A mutation identified in the present study. The p.G531E mutant protein in patient plasma is detectable with anti-FXII antibodies, however, its procoagulant activity is reduced. The presence of a circulating but dysfunctional coagulation factor protein variant is referred to as cross-reactive material (CRM)positive [5]. In the present study, we could not obtain a feline reactive anti-FXII antibody thereby precluding direct measurement of the amount of plasma FXII antigen in circulation. However, secretion of the p.G544A mutant rfXII protein in the culture medium supernatant was significantly decreased compared with wild type rfXII protein. The novel mutation is therefore predicted to result in CRM-negative deficient plasma.

In human populations, plasma FXII activity and antigen of Asian subjects were lower than those of Caucasians [4], and its cause is a genetic polymorphism $(46 \mathrm{C} / \mathrm{T})$ which leads to low translation of the $F 12$ gene [6]. In cats, the previously reported mutation, c.1321delC, was identified in United State, but was not observed in the present study. This difference suggests a geographical variation in distribution of feline F12 mutations. Additionally, the previously reported feline FXII mutation [1] and the c.1631G $>\mathrm{C}$ mutation of the present study were found in DSH cats. A survey of Feline FXII deficiency in the United States revealed that the trait was most commonly found in DSH cats, but that Siamese cats were apparently overrepresented in the study population [2]. Further studies are required to better characterize the geographical distribution and breed prevalence of feline FXII deficiency.

Factor XII deficiency, because of a lack of a bleeding tendency, is generally considered an incidental clinical finding. However, recent animal model studies suggest that FXII deficiency or inhibition of FXII activity contributes to a resistance to thrombus formation in the absence of abnormal bleeding [8, 10, 12]. As a result, FXII has been proposed to promote pathologic thrombosis, yet is not required for normal blood clot formation. In cats, thrombotic complications are recognized secondary to several diseases including cardiac, neoplastic and inflammatory disorders [3]. However, it remains to be investigated whether feline FXII deficiency has any protective benefit in preventing thrombotic events or improving survival.

In conclusion, we identified the p.G544A substitution as a novel mutation in exon 13 of the F12 gene coding for a mutant FXII protein that is retained intracellularly with minimal secretion into culture media. Cats homozygous for this mutation have less than $10 \%$ of normal FXII activity, whereas heterozygotes have more moderate FXII deficiency. These results, together with the previously reported feline $F 12$ mutation, provide the basis for comparative molecular analyses and epidemiologic studies of feline FXII deficiency to further clarify FXII's in vivo role in hemostatic and thrombotic processes.

ACKNOWLEDGMENTS. The authors are grateful to Dr. Marjory Brooks, College of Veterinary Medicine, Cornell University, for giving advice and carefully proofreading the manuscript. We also thank to Dr. Hiroshi Sugiya, Dr. Ken Okabayashi and Dr. Takanori Narita, Department of Veterinary Medicine, Nihon University, for their great help in western blot analysis.

\section{REFERENCES}

1. Bender, D. E., Kloos, M. T., Pontius, J. U., Hinsdale, M. E. and Bellinger, D. A. 2015. Molecular characterization of cat factor XII gene and identification of a mutation causing factor XII deficiency in a domestic shorthair cat colony. Vet. Pathol. 52: 312-320. [Medline] [CrossRef]

2. Brooks, M. and DeWilde, L. 2006. Feline factor XII deficiency. Compend. Contin. Educ. Pract. Vet. 28: 148-156.

3. de Laforcade, A. 2012. Diseases associated with thrombosis. Top. Companion Anim. Med. 27: 59-64. [Medline] [CrossRef]

4. Gordon, E. M., Donaldson, V. H., Saito, H., Su, E. and Ratnoff, O. D. 1981. Reduced titers of Hageman factor (factor XII) in Orientals. Ann. Intern. Med. 95: 697-700. [Medline] [CrossRef]

5. Iijima, K., Arakawa, Y., Sugahara, Y., Matsushita, M., Moriguchi, Y., Shimohiro, H. and Nakagawa, M. 2011. Factor XII Osaka: abnormal factor XII with partially defective prekallikrein cleavage activity. Thromb. Haemost. 105: 473-478. [Medline] [CrossRef]

6. Kanaji, T., Okamura, T., Osaki, K., Kuroiwa, M., Shimoda, K., Hamasaki, N. and Niho, Y. 1998. A common genetic polymorphism (46 C to T substitution) in the $5^{\prime}$-untranslated region of the coagulation factor XII gene is associated with low translation efficiency and decrease in plasma factor XII level. Blood 91: 2010-2014. [Medline]

7. Kier, A. B., Bresnahan, J. F., White, F. J. and Wagner, J. E. 1980. The inheritance pattern of factor XII (Hageman) deficiency in domestic cats. Can. J. Comp. Med. 44: 309-314. [Medline]

8. Matafonov, A., Leung, P. Y., Gailani, A. E., Grach, S. L., Puy, C., Cheng, Q., Sun, M. F., McCarty, O. J., Tucker, E. I., Kataoka, H., Renné, T., Morrissey, J. H., Gruber, A. and Gailani, D. 2014. Factor XII inhibition reduces thrombus formation in a primate thrombosis model. Blood 123: 1739-1746. [Medline] [CrossRef]

9. Mischke, R. 2012. Haemostasis: diagnostic techniques. pp. 189-200. In: BSAVA Manual of Canine and Feline Haematology and Transfusion Medicine, 2nd ed. (Day, M. J. and Kohn, B. eds.), BSAVA, Gloucester.

10. Müller, F., Mutch, N. J., Schenk, W. A., Smith, S. A., Esterl, L., Spronk, H. M., Schmidbauer, S., Gahl, W. A., Morrissey, J. H. and Renné, T. 2009. Platelet polyphosphates are proinflammatory and procoagulant mediators in vivo. Cell 139: 1143-1156. [Medline] [CrossRef]

11. Renné, T., Schmaier, A. H., Nickel, K. F., Blombäck, M. and Maas, C. 2012. In vivo roles of factor XII. Blood 120: 4296-4303. [Medline] [CrossRef] 
12. Renné, T., Pozgajová, M., Grüner, S., Schuh, K., Pauer, H. U., Burfeind, P., Gailani, D. and Nieswandt, B. 2005. Defective thrombus formation in mice lacking coagulation factor XII. J. Exp. Med. 202: 271-281. [Medline] [CrossRef]

13. Stavrou, E. and Schmaier, A. H. 2010. Factor XII: what does it contribute to our understanding of the physiology and pathophysiology of hemostasis \& thrombosis. Thromb. Res. 125: 210-215. [Medline] [CrossRef] 